\title{
Creación de significado online: recoger las voces de los y las fans de series televisivas
}

\author{
Online construction of meaning: capturing the voices \\ of $T V$ series fans
}

\author{
IOLANDA TORTAJADA \\ Universitat Rovira i Virgili \\ iolanda.tortajada@urv.cat (ESPAÑA) \\ Cilia Willem \\ Universidad Rovira i Virgili \\ cilia.willem@urv.cat (ESPAÑA)
}

Recibido: 25.012018

Aceptado: 07.11.2018

\section{RESUMEN}

Quienes siguen series televisivas, en ocasiones, comparten sus opiniones a través de espacios online más o menos institucionalizados. Estas prácticas nos permiten acceder a un conjunto de discursos e interacciones que, si bien no han sido inducidos en un contexto de investigación, proporcionan una gran cantidad de información sobre las lecturas y las apropiaciones que hacen fans y detractores de estos productos mediáticos. A partir de los comentarios expresados sobre Girls (HBO/Canal Plus, 2012-2017) en seis blogs diferentes (FormulaTV, Vertele, Jenesaipop, Blogelpaís, Foro Vandal y Foro Vogue), se explora la utilidad metodológica de los foros online en los estudios de recepción, problematizando el modelo desarrollado por Hall (1973). Girls ironiza sobre el día a día de cuatro jóvenes urbanitas neoyorquinas valiéndose para ello de marcos posfeministas, marcos contestatarios con el posfeminismo y marcos feministas (Bell 2013, Daalmans 2013, Fuller y Driscoll 2015, Weitz 2016). En línea con la riqueza narrativa de la serie, el contenido elaborado en estos entornos indica una notable complejidad de las lecturas expresadas en ellos. En nuestro estudio hemos puesto de manifiesto que, trabajando sobre el material recopilado en estos entornos online, afloran los diferentes tipos de lectura realizados y puede determinarse a qué clase de representaciones se asocia cada tipo de lectura, así como 
las estrategias establecidas para acercarse y alejarse de los presupuestos de la serie según las experiencias y los valores propios. Además, las interpretaciones vertidas permiten explorar tanto la vinculación que establecen fans y detractores entre sus experiencias cotidianas y las vivencias de los personajes de la serie como sus críticas a la cultura popular. Sin embargo, es imposible profundizar en estos significados sin combinar el análisis de los foros online con entrevistas en profundidad o grupos de discusión. Este tipo de metodologías, pues, más que para conocer a un colectivo o a las personas concretas y sus motivos, sirven para comprender los significados construidos acerca de un producto o manifestación cultural por parte de la audiencia activa. Por ello, en este trabajo se abordan las ventajas y limitaciones, tanto metodológicas como éticas, de este tipo de recogida y análisis de datos.

\section{PALABRAS CLAVE} tivo.

Girls, series TV, posfeminismo, foros online, análisis de contenido cualita-

\section{ABSTRACT}

Television series fans often share their opinions on - more or less institutionalized - online spaces. These spaces and practices allow us to access a set of discourses and interactions that, although they have not been induced in a research context, provide a large amount of information about the readings and the appropriation of meaning of fans and detractors. Using fan discussions about Girls (HBO/Canal Plus, 2012-2017) in six different Spanish blogs (FormulaTV, Vertele, Jenesaipop, Blogelpaís, Foro Vandal and Foro Vogue), we explore the methodological usefulness of online fora in reception studies, problematizing the model developed by Hall (1973). Girls is a satire evolving around the daily lives of four young New York urbanites, displaying post-feminist representation patterns, as well as critical and feminist ones (Bell 2013, Daalmans 2013, Fuller and Driscoll 2015, Weitz 2016). In line with the narrative richness of the series, the contents created on online fora indicate a remarkable complexity of the readings expressed by viewers. In our study we show that by working with the material collected from online environments, the different types of readings come to light and it can be determined to what kind of representations each type of reading is associated, as well as the strategies established to approach or move away from the series' presuppositions, according to viewers' own experiences and values. In addition, the discussions allow exploring both the connection established by fans and detractors between their daily experiences and the experiences of the characters in the series, and their critique of popular culture. However, we are aware that it is impossible to delve into these meanings without combining the analysis of the online fora with indepth interviews or discussion groups. 
This type of methodologies, then, rather than gaining deep knowledge about a collective or a specific segment of the population and their motives, help us to understand the meanings constructed about a product or cultural manifestation by the (active) audience. In this work we therefore address the advantages and limitations, both methodological and ethical, of this type of data collection and analysis.

\section{KEY WORDS}

Girls, TV show, post-feminism, online forums, qualitative content analysis.

\section{LA CREACIÓN DE SIGNIFICADO EN LOS ENTORNOS ONLINE}

Nuestra aproximación a las interacciones relacionadas con la recepción de series televisivas que se producen en los entornos online se sostiene en dos premisas: que las personas tienen competencias lingüísticas y comunicativas, por lo que son capaces de interactuar a través del diálogo (Habermas 1987) y que cualquier influencia de los medios depende de la manera en las personas recogen y utilizan sus contenidos, que son filtrados contextualmente e interpretados antes de reflejarse en la experiencia o en la conducta (Blumer 1982).

Por ello, las prácticas online, como otros consumos mediáticos, están vinculadas a la creación de significado. Dicho de otra forma, sólo desde la creación de significado existen y se sostienen. Esta constatación proviene del trabajo desarrollado por los autores y autoras que, bajo el paraguas de los Estudios Culturales, establecieron que la recepción consiste en una apropiación activa del contenido mediático. Y, como sostiene Livingstone (2004), la misma agencia, heterogeneidad y resistencia que presuponemos en las interpretaciones y apropiaciones que llevan a cabo las audiencias, puede aplicarse a las prácticas online. Así, las conversaciones en entornos online dan cuenta de varios procesos de recepción, los que tienen que ver propiamente con las series y los que tienen que ver con la creación de contenidos digitales sobre las series.

Entre otras dimensiones, Internet debe ser visto también como un espacio para la construcción social de las identidades y de las comunidades. Los procesos de negociación y construcción identitaria se hacen visibles, en gran medida, a través de los textos que se comparten en estos entornos. Dichos textos son la base y la principal característica del tipo de interacciones en la red de las que se ocupa este estudio, aquellas que se sostienen en la escritura y en la complicidad de una producción veraz (Markham 2004). La web ofrece múltiples espacios en los que nos hacemos existir mediante lo que allí escribimos (boyd 2006) y en los que las diferentes subculturas y comunidades encuentran nuevas formas de conexión (Banet-Weiser et al. 2014).

Estas prácticas, que podemos definir, a grandes rasgos, como participaciones en una conversación, implican el uso de numerosos canales en los que las au- 
diencias reconfiguran la lectura preferente de los contenidos de los medios y en los que crean nuevos significados a través de textos mediáticos. En este sentido, las audiencias están siempre participando, y las conversaciones que mantienen van más allá de los contenidos televisivos que las han unido en primera instancia, por lo que, en sus interacciones, construyen comunidad. Así, la creatividad debe entenderse como algo que tiene que ver con la creación de identidades, relaciones y comunidades (Banet-Weiser et al. 2014).

En los entornos web 2.0, la voz de las audiencias se hace visible (Hermes 2009). Esto no significa que la creación de significado sea algo sencillo o que los espacios en los que las audiencias interactúan estén libres de influencias o dinámicas industriales. Existe el riesgo de que las reapropiaciones expresadas por fans y audiencias sean explotadas comercialmente, de que la creatividad de sus interpretaciones se convierta en un valor de producción y de que, en un cierto sentido, la propia participación acabe articulándose a través de las estrategias de márketing empresariales (Andrejevic 2008, Banet-Weiser et al. 2014, Hermes 2009), a la vez que la implicación con los diversos textos a los que nos enfrentamos cotidianamente es muy diversa (Hermes 2009) y que las motivaciones para participar pueden ser múltiples, y no necesariamente responder a la implicación en la comunidad sino a la retirada del mundo (Andrejevic 2008). Ser una audiencia activa requiere trabajo y puede que no todo el mundo esté dispuesto a involucrarse (Banet-Weiser et al. 2014).

Aún así, numerosos estudios (ver, entre otros muchos, los trabajos de boyd o Livingstone) muestran que la participación en los entornos online es compleja y contradictoria y que comprenderla supone dejar de lado presupuestos simplistas como el de que la interactividad es intrínseca al medio (Jenkins 2009) o el uso de ejes explicativos como el de producción-consumo o el de producción comercialproducción popular, que no son suficientes para capturar la sofisticación de las prácticas que se producen en la red porque van mucho más allá de los binarismos que establecen dichos ejes (Banet-Weiser et al. 2014). Parece oportuno preguntarse por el tipo de oportunidades para la interacción que ofrece Internet, por la clase de participaciones que allí se producen y por cómo la participación es leída por quienes interactúan en estos espacios. En definitiva, se trata de recoger y hacer visibles las interpretaciones, las identificaciones, los placeres, el activismo y la creatividad que manifiestan quienes siguen las series, así como la creación conjunta de significado que se produce al compartir, de forma seriamente lúdica, todo este universo.

\section{LAS APROPIACIONES DEL CONTENIDO TELEVISIVO}

La creación de sentido es un proceso complejo que se produce en diversos espacios, a lo largo del tiempo, y que, por definición, es cambiante. En el ámbito de los estudios de recepción, el trabajo que se toma como referencia es el de Stuart Hall (1973), quien planteó la condición abierta y, al mismo tiempo, estructurada de los textos y la capacidad de las audiencias para interpretarlos, 
ya fuera en el sentido en el que pretendía quien los emitió, ya fuera en cualquier otro. Esto dio lugar a la conocida tipología de las categorías de lectura dominante, negociada y oposicional, siendo la primera la que se refiere a la asunción del mensaje según ha sido definido preferentemente por el emisor (de acuerdo a los valores hegemónicos), la segunda la que tiene que ver con la adopción parcial del discurso propuesto, mientras que se descartan algunos de sus aspectos, y la tercera con el rechazo (resistente o subversivo) del contenido emitido.

En el desarrollo de diversas investigaciones, se han constatado algunas de las limitaciones de esta propuesta de análisis definida por Hall (1973). El propio Morley (1996), cuando puso a prueba el modelo, determinó que aspectos como la clase social, el género o las relaciones de poder en la familia jugaban un papel importante en el tipo de lectura y que el consumo tendía a ser grupal, más que individual, por lo que la categorización establecida por Hall debía complejizarse. Otra contribución fue la de Hacker et al. (1991) que dividieron en subcategorías la noción de lectura oposicional para referirse a los diversos grados en los cuales una crítica puede ser planteada (el simple rechazo, la falta de credibilidad, generando un argumento alternativo o ejerciendo una resistencia ideológica). Esto no quiere decir que las lecturas más oposicionales supongan necesariamente un desafío a los estereotipos o un posicionamiento progresista (Buckingham 1993, Ging 2005). Finalmente, y por lo que se refiere a la adopción del mensaje, Palmer y Hafen (1999) elaboraron el concepto de lectura no oposicional distinguiendo entre aceptaciones ingenuas y sofisticadas.

Por otra parte, las investigaciones de Lozano y Frankenberg (2011) mostraron que pueden producirse lecturas oposicionales de textos cuyo contenido es crítico, poniendo en evidencia la complejidad y mutabilidad de las categorías. Por ello, y para aplicar este instrumento, hay que tener en cuenta los componentes hegemónicos del texto que va a ser leído, los grados de aceptación y crítica en las lecturas manifestadas y los factores estructurales y contextuales del consumo y preguntarse por la forma en la que las experiencias personales y los discursos mediáticos se van entrelazando en un flujo de identificaciones y contestaciones (Moran 2003, Price 1998).

Girls (HBO/Canal Plus, 2012-2017) es un dramedy creado y protagonizado por Lena Dunham y producido por Judd Apatow que, durante 6 temporadas y 62 capítulos, nos introduce en las vidas y las relaciones afectivas de cuatro veinteñeras que viven en Nueva York. La serie, que ha sido premiada en diversas ocasiones y ha recibido el interés y los halagos de la crítica, responde al paradigma de la televisión de calidad por sus guiones arriesgados, los elementos rupturistas, la originalidad y la creatividad en lo técnico y lo narrativo (Menéndez y Zurian 2014). Girls presenta una versión sutilmente provocadora del feminismo en un momento en el que la cultura popular no puede dar cuenta de las reivindicaciones feministas si no es para rechazarlas mientras, aparentemente, las abraza (Tortajada y Van Bauwel 2012, Fuller y Driscoll 2015). El concepto de posfeminismo, tal como lo entendemos aquí, nos sirve para dar cuenta de las transformaciones en las representaciones de las feminidades y las masculinidades. Unas caracterizaciones que se sostienen en la falsa premisa de que el feminismo ha 
conseguido sus propósitos. Así, las reivindicaciones feministas se relegan al pasado, a la vez que se reclaman, para aparecer sin contenido político ninguno: la igualdad, el empoderamiento, el cuerpo o el deseo sexual son reclamados desde la estética y el consumismo porque ¿qué importa ya si ya tenemos todo lo que queríamos? (ver, entre otras, McRobbie 2004, Gill 2007b, Lazar 2009, FiguerasMaz, Tortajada y Willem 2017).

Como es habitual en los medios y en la sociedad, en Girls se entrelazan de forma aproblemática los marcos feministas y sus versiones posfeministas despolitizadas. Por una parte, la serie retrata unas mujeres que no necesariamente responden a los patrones de belleza canónica, que fracasan en lo laboral y que tienen problemas para gestionar tanto sus relaciones amorosas como la supuesta amistad que las une. Por otra parte, las protagonistas expresan y satisfacen su deseo sexual sin un ápice de culpa, toman sus propias decisiones y asumen sus fracasos sin que sus identidades o expectativas se vean dañadas. El relato es ambiguo y, en ocasiones, no sólo parece cuestionar los patrones representacionales posfeministas sino que apenas se detectan posturas antifeministas (Menéndez 2017), pero debido a los múltiples giros y contradicciones en todo aquello que se retrata (solidaridad femenina, igualdad, libertad sexual, éxito, etc.), podemos afirmar que la serie toma una perspectiva posfeminista (Bell 2013, Daalmans 2013; Figueras-Maz, Tortajada y Willem 2017), impulsando un discurso repleto de ironías y ambigüedades (Weitz 2016) y un panorama desolado en el que, ni siquiera la celebración del neoliberalismo que está en la base del propio posfeminismo, se salva (Menéndez 2017).

Así pues, reconocer la agencia de las audiencias nos conduce a reflexionar sobre el tipo de preguntas de investigación que van a resultar más pertinentes $y$, en este sentido, compartimos el enfoque de Bird (2010) cuando propone que hay que ir más allá de interesarse por lo que la gente dice o hace en relación a los medios para sumergirse en las múltiples formas en las que éstos se incorporan en las prácticas comunicativas y culturales cotidianas. La idea de la práctica debe de estar en el centro de los estudios y de la conceptualización de las audiencias mediáticas.

\section{INTERNET COMO HERRAMIENTA METODOLÓGICA EN LOS ESTUDIOS DE RECEPCIÓN}

Internet es, al mismo tiempo, una herramienta de investigación y un objeto de estudio valioso (Markham 2004). Según Lotz y Ross (2004), la red ha supuesto la principal revolución en los estudios de recepción desde que estos fueron reivindicados y constituidos por la corriente teórica de los estudios culturales. Tal como apuntaba Ardèvol (2012), a partir de los 90, las primeras investigaciones 'etnográficas' de la comunicación en internet abrieron paso a la validación y legitimización de su estudio desde un punto de vista social y cultural. Cuando nos fijamos concretamente en los foros de fans, vemos que los y las seguidoras de series de televisión - u otros productos de cultura popular - adop- 
taron rápidamente la tecnología digital y crearon espacios digitales de encuentro (Jenkins 2009) para allí comentar, criticar, y crear nuevos contenidos relacionados con sus programas favoritos (ver Baym 1993, 1999 con sus ejemplos de las primeras comunidades de seguidores de soap operas).

En este trabajo nos interesan sobre todo los foros de fans como herramienta metodológica nueva de los clásicos estudios de recepción, en este caso concreto para acercarnos a las lecturas que hacen los y las seguidoras españolas de la serie Girls: cómo interpretan sus contenidos, cómo critican a la creadora de la serie, Lena Dunham, y qué comentan sobre los temas que aparecen en la serie, como el sexo, la amistad, el éxito profesional y el consumo. Los resultados del estudio fueron publicados recientemente (Figueras-Maz, Tortajada y Willem 2017), y muestran cómo el análisis de los comentarios de fans y detractores españoles de Girls nos sirvió para captar la complejidad de las lecturas que realizaban. Observamos que tanto las lecturas preferentes como las oposicionales (Hall 1973) podían implicar un alineamiento con el posfeminismo, o todo lo contrario. En el artículo se pone de manifiesto que los y las seguidores de la serie, en su mayoría, realizan lecturas preferentes en aquellos momentos en los que la serie está redibujando un marco de representación posfeminista. A la vez, Girls muestra el personaje de Hannah, que - lejos de responder a los cánones tradicionales de belleza y trabajar para tener un cuerpo perfecto - capítulo tras capítulo nos muestra todas sus imperfecciones, lo poco que le preocupan, y lo mucho que disfruta de su cuerpo y de sus relaciones. Esto genera lecturas preferentes y lecturas oposicionales.

Si bien la herramienta metodológica de análisis de los foros no nos dice nada sobre sus participantes como personas o siquiera como comunidad, sí lo hace como miembros de una audiencia activa. Es decir, desde el punto de vista del interaccionismo simbólico (Blumer 1982), esta herramienta nos puede servir para dibujar un panorama claro sobre la recepción de un producto cultural concreto, como es una serie de televisión, en un contexto cultural concreto (en este caso, España).

\section{ESTUDIAR LOS FOROS EN INTERNET: VENTAJAS Y LIMITACIONES}

Como hemos venido indicando, las ventajas de estudiar la recepción de una serie a través de su audiencia activa online (foros de fans) son múltiples. A nivel práctico, la recogida de datos es relativamente fácil y eficiente: buscar y recoger las voces de la audiencia, independientemente de su ubicación geográfica, se hace mediante la simple bajada y archivado del texto completo de uno o varios foros seleccionados para responder a las preguntas de investigación. Una vez guardados como archivo, el material ya se puede tratar como un texto normal, aplicando diferentes técnicas analíticas, según la necesidad concreta, como por ejemplo análisis (crítico) de discurso, análisis de contenido cuantitativo y/o cualitativo, análisis conversacional, etc... 
Sin embargo, tanto a nivel metodológico como a nivel ético hay una serie de limitaciones o problemas en el acceso a y el análisis de este tipo de datos, que hacen que sea más complejo de lo que parece. A nivel metodológico, nos encontramos principalmente con un desconocimiento sobre la muestra: por un lado, un foro (o varios foros) no puede ser nunca representativo de ningún tipo de población y, por lo tanto, los resultados no se pueden extrapolar. Por otro lado, como investigadoras no conocemos la identidad real de las y los participantes, sólo sus alias o nombres de usuarios y lo que ellos mismos han elegido mostrar o informar en sus perfiles. En sí esto no es un problema, pero dependiendo de qué estamos estudiando y con qué objetivo (por ejemplo conocer a fondo el perfil de las personas concretas que están activas en el foro), puede ser importante saber el sexo, la edad, o la ubicación geográfica de las y los fans de una serie. En tal caso, la simple bajada de los textos del foro no será del todo suficiente. Relacionado con esto, contactar con estas personas - por ejemplo para hacer entrevistas en profundidad - es extremadamente difícil, debido a que sus nombres pueden ser ficticios, y los datos de contacto normalmente no son públicos. En nuestro caso, teniendo como objeto de estudio la serie Girls y cómo sus fans la interpretan, fue suficiente analizar los foros a nivel textual. Una limitación de este estudio fue, pues, que no pudimos profundizar en estos significados si no completábamos el análisis desarrollado con entrevistas o grupos de discusión.

Otras limitaciones a nivel metodológico de una mera recogida de estos foros (es decir, sin intervención del investigador como participante) son la parcialidad de las voces recogidas, el tamaño del mensaje - que muchas veces consiste en una o algunas frases y puede dar lugar a interpretaciones por su brevedad - y el hecho de que no haya diálogo con los participantes, por lo tanto no se puede averiguar la veracidad de lo que dicen.

A nivel ético, cuando analizamos foros en internet, deben tomarse una serie de decisiones acerca de la intrusión que puede suponer la participación del investigador o investigadora en los foros y velar por el anonimato de quienes forman parte de la investigación. En primer lugar, como en cualquier otra investigación, y aunque no siempre es fácil, o a veces es imposible, hay que intentar obtener su consentimiento (Lotz y Ross 2004). A pesar de que la mayoría de los foros en internet son públicos, sus miembros pueden percibir sus interacciones como privadas (Markham 2004). Otros grupos son conscientes de que sus comunicaciones son públicas, pero no quieren ser objeto de estudio (Hudson y Bruckman 2002). En segundo lugar, el equipo investigador tendrá que decidir si se va a limitar a 'observar' (es decir, sólo analizar lo que se dice en esos foros, sea en directo o a posteriori), o en cambio va a intervenir como participantes en la conversación. La acción investigativa se puede hacer de modo anónimo, o revelando el estatus de investigador/a, escogiendo una de las modalidades de la observación participante que proponen Wester, Renckstorf y Scheepers (2006) para cualquier investigación etnográfica: observación anónima (modo 'fly-on-the-wall'), observación y/o participación con revelación del estatus de investigador, o participación anónima (modo 'infiltración'). Está claro que cada una de estas modalidades tiene sus ventajas e inconvenientes a nivel metodológico, pero a nivel ético las 
opciones anónimas son las más problemáticas según las tendencias actuales de las investigaciones sociológicas (Markham 2004, Wester et al. 2006). En nuestro caso, el objetivo de la investigación era averiguar cómo las y los fans de Girls interpretaban la serie, por lo que simplemente fuimos observadoras a posteriori, por lo que consideramos que no hacía falta revelar nuestro estatus de investigadoras en los foros estudiados.

En conclusión, la reflexión y la crítica deberían ser una constante en el diseño y desarrollo de la investigación, así como el uso de diferentes técnicas de recogida de datos (Lotz y Ross 2004).

\section{ANÁLISIS CUALITATIVO DE CONTENIDOS DE LOS FOROS EN INTERNET}

Los blogs españoles seleccionados como muestra para la investigación fueron aquellos que contenían un foro específico para hablar de la serie y en los que se generó un mayor número de comentarios. Se analizaron todos los comentarios realizados en los foros desde el inicio de la emisión de la serie en España (2012) hasta octubre de 2016 (5 temporadas):

FormulaTv (www.formulatv.com), portal especializado en televisión que, entre otros servicios, da acceso a datos de audiencia y que dispone de una gran comunidad de usuarios en español a través de diversas opciones de participación, entre ellas foros.

Vertele (www.vertele.com), portal sobre el mundo de la televisión en España donde los periodistas especializados cubren diversos aspectos de la actualidad televisiva, entre los cuales se encuentra el análisis de audiencias, y en el que los usuarios y usuarias pueden participar con sus comentarios.

Jenesaispop (www.jenesaispop.com), es un sitio web español fundado por un grupo de periodistas que ofrece noticias sobre la actualidad musical independiente, críticas de discos, información sobre conciertos y sobre televisión (entre otras opciones) y que permite a sus usuarios e usuarias registrarse en los diversos foros del sitio.

Blog de El País (http://cultura.elpais.com/cultura/2013/02/14/television/13608253 20_136082.html), blog sobre televisión y series. En este caso se analizaron los comentarios generados a partir del post Girls: ¿sí o no?.

Foro (en) Vandal (www.forovandal.net), Vandal es un sitio web de noticias sobre videojuegos y tecnología que incluye foros sobre estos temas y uno específico sobre cine y televisión.

Foro Vogue (www.foros.vogue.es/viewforu m.php?f=66), foro titulado "Debate abierto" de la revista Vogue sobre ocio y cultura.

Cuando accedimos a estos foros, el estudio de recepción de la serie Girls partía de una metodología cualitativa, empleada en otros estudios realizados con datos extraídos de foros de fans (ver Baym, 1999; Buckingham y Rodríguez, 2013; Masanet y Buckingham, 2015). En un primer momento, los comentarios de fans y detractores que hacían referencia a estos temas fueron extraídos directamente de los foros y compilados en un documento según las categorías de 
análisis iniciales, definidas en el marco teórico. Uno de los ejes principales del análisis consistió en determinar el tipo de lectura de fans y detractores de la serie basado en Hall (1973): (a) lectura preferente o dominante, (b) lectura negociada, (c) lectura oposicional. El otro eje de análisis se centró en las características del posfeminismo derivadas del apartado teórico: autenticidad, autorregulación, agencia sexual/libertad de elección (ver el artículo original, Figueras-Maz, Tortajada y Willem 2017).

Una vez recopilados todos los fragmentos de texto relevantes extraídos de los foros, pasamos a tratar el material como un texto único a analizar mediante un análisis de contenido cualitativo con método inductivo de construcción de categorías (Mayring 2004). A diferencia del clásico análisis de contenido, que principalmente consiste en registrar meras ocurrencias o frecuencias en un producto mediático (y por lo tanto es cuantitativo por definición), el análisis de contenido cualitativo se base en la construcción y codificación sistemáticas de categorías, no necesariamente pre-establecidas. En nuestro caso utilizamos un análisis mixto, combinando una codificación del material según categorías pre-establecidas por el marco teórico, por un lado, y generadas por el propio material, por otro, lo que Mayring (2004: 268) llama "generación inductiva de categorías". Para este estudio de los foros de Girls, una investigadora del equipo elaboró una primera taxonomía descriptiva que se fue confeccionando progresivamente y validando (o descartando) categorías a través de sucesivas lecturas por parte de las otras dos investigadoras. El proceso se monitorizó a través de mecanismos de fiabilidad inter-codificadores, hasta establecer la taxonomía de categorías completa y volver a aplicarla al conjunto del material.

Este tipo de procedimientos obliga a quien investiga a tener en cuenta la generación de nuevas categorías o conceptos durante el proceso de codificación, así como la eliminación de categorías si resulta que no tienen ninguna ocurrencia o relevancia para el material. En nuestro caso, por ejemplo, pre-establecimos la categoría "empoderamiento falso" - procedente del marco teórico relativo al posfeminismo - que después de una primera ronda de codificación por parte de las investigadores fue eliminada visto que ningún fragmento del material fue asignada a esa categoría. Por el contrario, el equipo investigador no anticipó la categoría "amor-odio a Lena Dunham" (Durham es productora de la serie y intérprete de su personaje principal, Hannah), que resultó ser una de las más codificadas en todo el texto.

\section{DISCUSIÓN Y CONCLUSIONES}

A pesar de que los foros online sobre Girls que hemos analizado fueron creados en portales dedicados a la información televisiva o en páginas web de los propios medios de comunicación, quienes siguieron o abandonaron la serie aprovecharon esos espacios para opinar libremente dentro del margen permitido por los responsables de la moderación de los foros. Pudimos recoger abundante material de cuyo análisis se desprenden algunos aspectos para la reflexión. Ex- 
cepto en casos puntuales de contraposición de opiniones, son mínimas las interacciones o conversaciones entre participantes; la mayoría de mensajes abordan la serie desde un punto de vista personal, pero sin dar respuesta a otros mensajes. En este caso, pues, no podemos afirmar que los foros online hayan generado comunidad (Banet-Weiser et al. 2014).

En cuanto al tipo de interpretaciones y lecturas que realizan quienes siguen Girls, el análisis de los foros nos ha permitido captar que la relación que se establece con la serie y con sus protagonistas se expresa con términos absolutos, ya sea de adoración (la mayoría), ya sea de rechazo. Sin preguntar a quienes participan en los foros, no podemos saber si es la propia presencia y actividad en los foros la que provoca este tipo de aseveraciones o se debe a la serie o una una combinación de ambas cosas, pero, en todo caso, las opiniones sobre la serie son taxativas. Al mismo tiempo, la perspectiva posfeminista que enmarca las tramas de la serie (Bell 2013, Daalmans 2013) es aceptada, aunque los textos generados online de quienes siguen Girls evidencian diversas estrategias para acercarse y alejarse de los presupuestos de la serie según las experiencias y los valores propios (Figueras-Maz, Tortajada y Willem 2017).

La ironía y la ambigüedad de la serie (Weitz 2016) provoca lecturas literales, pero también sofisticadas (Palmer y Hafen 1999), especialmente por lo que se refiere a la autenticidad, que es uno de los ejes fundamentales de la serie (Figueras-Maz, Tortajada y Willem 2017). La autenticidad se presenta vinculada a las relaciones sexuales y afectivas, a la precariedad laboral y a la supervivencia en la ciudad y esto provoca numerosas identificaciones entre quienes siguen la serie (a la vez que es el motivo principal por el que algunas personas manifiestan en los foros su decisión de dejar de ver la serie). Así, la mayoría de textos defienden la idea de que la serie es auténtica y de que su protagonista es una rebelde, al mostrar sin pudor sus propios fracasos y su falta de estrategias para salir adelante. Esta ambivalencia del fracaso entendido como éxito es uno de los patrones de representación posfeminista que se ha ido consolidando en diversos medios (Caballero, Tortajada y Willem 2017) y que es leído de forma preferente, no de manera ingenua, sino sofisticada, a través de la conexión con las vivencias cotidianas (Palmer y Hafen 1999). Otros aspectos que quienes siguen la serie asocian a la autenticidad son los personajes masculinos que son vistos como un cuestionamiento de las masculinidades hegemónicas (Hanke 1990) o la ruptura con los estereotipos.

Si bien no podemos considerar que las prácticas online se han constituido como conversaciones o han servido para construir comunidad, en algunas ocasiones, hemos constatado que quienes han participado en los foros de Girls llevaban sus discursos más allá de la serie, por ejemplo, al criticar las representaciones mainstream del cuerpo, justificando que la protagonista principal de la serie muestre su cuerpo (y reivindique el derecho a hacerlo), a pesar de que éste no responde a los cánones de belleza tradicionales y que la serie, en palabras de quienes la siguen, tenga un halo de feísmo. Las interpretaciones sobre la serie incorporan la explotación de la imperfección que propone Girls y muestran, por un lado, la vinculación de las experiencias cotidianas que quienes participan en los 
foros establecen con las vivencias de los personajes de la serie y, por otro lado, sus críticas personales a la cultura popular, que presenta imágenes estrechas y cosificadoras del cuerpo. Frente a la perfección, se abraza el patetismo.

En un contexto posfeminista y neoliberal, las jóvenes tienen la obligación de ser 'independientes' y 'triunfar' en los ámbitos laboral y de las relaciones (Fuller y Driscoll 2015, McRobbie, 2007). Como hemos visto, Girls subvierte este postulado presentando una versión irónica y ambigua del fracaso y de las expectativas sociales y familiares acerca de lo que supone salir adelante con éxito, que es adoptada ampliamente por quienes siguen la serie. Igualmente, el acercamiento a los foros también nos ha permitido recoger y clasificar algunas expresiones de rechazo ingenuo (Palmer y Hafen 1999) y crítica sin argumentos (Hacker, Coste y Kamm 1991), como cuando alguien expresa su falta de interés por la vida de los personajes de la serie. Sin embargo, este distanciamiento es escaso y la mayoría de los textos manifiestan un compromiso emocional con la serie, calificando a su protagonista como heroína y defendiendo a los personajes a pesar de sus imperfecciones y sus errores, para los que se inventan todo tipo de justificaciones. Esta preponderancia de las lecturas preferentes puede deberse a que las personas a las que les gusta la serie se sienten más motivadas para participar en los foros.

En conclusión, a pesar de las limitaciones de los espacios online como objeto de estudio, que hemos descrito en detalle en este artículo, el acercamiento a los foros online nos ha permitido recoger con detalle la riqueza y la complejidad de las interpretaciones que se han realizado sobre Girls, así como la agencia en las prácticas online (Livingstone 2004). Este tipo de metodologías, pues, más que para conocer a un colectivo o a las personas concretas y sus motivos, sirven para comprender los significados construidos acerca de un producto o manifestación cultural por parte de la audiencia activa.

\section{BIBLIOGRAFIA}

ANDREJEVIC, M. (2008): "Watching Television Without Pity: The Productivity of Online Fans", Television \& New Media, 9(1), pp. 24-46.

ARDĖVOL, E. (2012): "Virtual/Visual Ethnography: Methodological Crossroads at the Intersection of Visual and Internet Research", en Advances in Visual Methodology, London, Sage, pp. 74-94.

BANET-WEISER, S., BAYM, N. K., COPPA, F., GAUNTLETT, D., GRAY, J. JENKINS, H. y SHAW, A. (2014): "Participations: Dialogues on the Participatory promise of Contemporary Culture and Politics", International Journal of Communication, 8, pp. 1069-1088.

BAYM, N. K. (1993): "Interpreting Soap Operas and Creating Community: Inside a Computer-Mediated Fan Culture", Journal of Folklore Research, 30(2/3), pp. 143176.

BAYM, N. K. (1999): Tune in, log on: Soaps, fandom, and online community. Thousand Oaks CA, Sage. 
BELL, K. (2013): “'Obvie, We're the Ladies!' Postfeminism, privilege, and HBO's newest Girls", Feminist Media Studies, 13(2), pp. 363-366.

BIRD, E. (2010): "From fan practice to mediated moments: the value of practice theory in the understanding of media audiences", en Theorising Media and Practice.,New York/Oxford, Berghahn Books, pp. 85-104.

BLUMER, H. (1982): El interaccionismo simbólico: perspectiva y método, Barcelona, Editorial Hora.

BUCKINGHAM, D. (1993): Children talking television: The making of television literacy, London, Falmer Press.

BOYD, DANAH. (2006): "Friends, Friendsters, and MySpace Top 8: Writing Community Into Being on Social Network Sites", First Monday, 11:12, December.

CABALlERO, A., TORTAJADA, I. y WILLEM, C. (2017): “Autenticidad, marca personal y agencia sexual: el posfeminismo lésbico en Youtube”, Revista de Investigaciones Feministas, 8(2), pp. 353-368.

DAALMANS, S. (2013): “'I'm Busy Trying to Become Who I Am': Self-entitlement and the city in HBO's Girls", Feminist Media Studies, 13(2), pp. 359-380.

FIGUERAS-MAZ, M., TORTAJADA, I. y WILLEM, C. (2017): "Patrones de representación posfeministas en Girls. Análisis de los significados construidos por fans y detractores", Oceánide, 9.

FULLER, S., y DRISCOLL, C. (2015): “HBO's Girls: gender, generation, and quality television", Continuum: Journal of Media \& Cultural Studies, 29(2), pp. 253-262.

GILL, R. (2007a): Gender and the Media, Cambridge, Polity.

GILL, R. (2007b): "Postfeminist media culture: Elements of a sensibility", European Journal of Cultural Studies, 10(2), pp. 147-166.

GILL, R. (2008): "Empowerment/Sexism: Figuring Female Sexual Agency in Contemporary Advertising", Feminism and Psychology, 18(1), pp. 35-60.

GING, D. (2005): “A 'Manual on Masculinity'? The consumption and use of mediated images of masculinity among teenage boys in Ireland", Irish Journal of Sociology, 14(2), pp. 29-52

HABERMAS, J. (1987): Teoría de la acción comunicativa I i II, Madrid, Taurus.

HACKER, K., COSTE, T.G., KAMM, D. y BYBEE, C. (1991): “Oppositional readings of network TV news", Discourse \& Society, 2(2), pp. 183-202.

HALL, S. (1973): "Encoding/decoding", en Culture, Media, Language: Working Papers in Cultural Studies 1972-79, London, Hutchinson, pp. 128-38.

HANKE, R. (1990): "Hegemonic masculinity in Thirty Something", Critical Studies in Mass Communication, 7(3), pp. 231-248.

HERMES, J. (2009): "Audience Studies 2.0. On the theory, politics and method of qualitative audience research", Interactions: Studies in Communication and Culture, 1(1), pp. 111-127.

HEWSON, C., YULE, P., LAURENT, D. y VOGEL, C. (2003): Internet Research Methods, London, Sage.

HUDSON, J., y BRUCKMAN, A. (2002): "IRC Français: The Creation of an Internetbased SLA Community", Computer Assisted Language Learning, 15(2), pp. 109134.

JENKINS, H. (2009): Fans, blogueros y videojuegos. La cultura de la colaboración, Barcelona, Paidós.

LAZAR, M. (2009): "Entitled to consume: Postfeminist femininity and a culture of postcritique", Discourse and Communication, 3(4), pp. 371-400. 
LIVINGSTONE, S. (2007): “The Challenge of Engaging Youth Online: Contrasting Producers' and Teenagers' Interpretations of Websites", European Journal of Communication, 22(2), pp. 165-184.

LOTZ, A. y ROSS, S. (2004): “Toward Ethical Cyberspace Audience Research: Strategies for Using the Internet for Television Audience Studies", Journal of Broadcasting \& Electronic Media, 48(3), pp. 501-513.

LOZANO, J. y FRANKENBERG, L. (2011): "Lecturas críticas y oposicionales de películas de Hollywood por jóvenes mexicanos: el caso de Ángeles y Demonios", Anagramas, 9(18), pp. 165-184.

MANN, C. y STEWART, F. (2000): Internet Communication and Qualitative Research: A Handbook for Researching Online, London, Routledge.

MARKHAM, A. (2004): "Internet communication as a tool for qualitative research", en Qualitative Research: Theory, Methods, and Practice, London, Sage, pp. 95-124.

MAYRING, P. (2004): "Qualitative Content Analysis", en A Companion to Qualitative Research, London, Sage, pp. 266-269.

McROBBIE, A. (2004): "Post-feminism and popular culture", Feminist Media Studies, 4(3), pp. 255-264.

McROBBIE, A. (2007): “Top Girls?”, Cultural Studies, 21(4-5), pp. 718-737.

MENÉNDEZ, I., y ZURIÁN, F. (2014): "Mujeres y hombres en la ficción televisiva norteamericana hoy", Anagramas: Rumbos y sentidos de la comunicación, 13(25),pp. 54-61.

MENÉNDEZ, I. (2017): "Entre el neomachismo y el retrosexismo: antifeminismo contemporáneo en las industrias culturales", Prisma Social: revista de investigación social, 2, pp. 1-30.

MORAN, K. (2003): "Reception analysis: Latina teenagers talk about telenovelas", Global Media Journal, 2(2).

MORLEY, D. (1996): Televisión, audiencias y estudios culturales, Buenos Aires, Amorrortu.

PALMER, A. y HAFEN, T. (1999): “American TV through the Eyes of German Teenagers", en Images of the U.S. around the World. A Multicultural Perspective, New York: State University of New York Press, pp. 135-146.

PINK, S. (2007): Doing Visual Ethnography: Images, Media and Representation in Research, London, Sage.

PRICE, S. (1998): Media Studies, Harlow, Longman.

TORTAJADA, I. y Van BAUWEL, S. (2012): "Gender and communication: contemporary research questions", Catalan Journal of Communication and Cultural Studies, 4(2), pp. 143-153.

WEITZ, R. (2016): “Feminism, Post-feminism, and Young Women's Reactions to Lena Dunham's Girls", Gender Issues, 33(3), pp. 218-234.

WESTER, F., RENCKSTORF, K. y SCHEEPERS, P. (2006): Onderzoekstypen in de communicatiewetenschap, Alphen aan de Rijn, Kluwer. 